\title{
Multiple epiphyseal dysplasia, with miniepiphyses
}

INSERM

\section{Source}

INSERM. (1999). Orphanet: an online rare disease and orphan drug data base. Multiple epiphyseal dysplasia, with miniepiphyses. ORPHA:166032

Multiple epiphyseal dysplasia, with miniepiphyses is a rare primary bone dysplasia disorder characterized by strikingly small secondary ossification centers (mini-epiphyses) in all or only some joints, resulting in severe bone dysplasia of the proximal femoral heads. Short stature, increased lumbar lordosis, genua vara and generalized joint laxity have also been reported. 